\title{
Atemwegsmanagement beim Kind: Notfalltechniken Schritt für Schritt
}

Nora Jahn, Sebastian G. Russo, Claudia Höhne

\section{Ziel und Zweck der Notfalltechnik}

Notfälle mit Kindern machen ca. 5-10\% aller präklinischen Notfälle aus. Invasive Maßnahmen zur Sicherung der Atemwege sind davon in weniger als $5 \%$ nötig $[1,2]$. Nicht zuletzt aufgrund der insgesamt geringen Inzidenz stellt die Atemwegssicherung bei Kindern daher für viele praktizierende Notfallmediziner und Rettungskräfte eine große Herausforderung dar. Es fehlt an der notwendigen Routine im Umgang mit kindlichen Atemwegen bei einer gleichzeitig hohen emotionalen Belastung.

Dieser Beitrag richtet sich daher weniger an notfallmedizinisches Personal mit einer ausgewiesenen Expertise im Bereich der kindlichen Atemwegssicherung. Vielmehr werden Schritt-für-Schritt-Techniken vorgestellt, die das oberste Ziel einer suffizienten Oxygenierung haben und i.d.R. auch für weniger erfahrene Kolleginnen und Kollegen mit einer hohen Erfolgsaussicht assoziiert sind.

\section{Indikationen, Techniken, Fallstricke und Tipps}

\section{Physiologie und Anatomie des kindlichen Atemwegs}

Sauerstoffmangel führt im Kindesalter rasch zu Bradykardie und Asystolie. Die Sicherstellung einer ausreichenden Oxygenierung und Ventilation steht daher im Rahmen der Notfallversorgung im Kindesalter an erster Stelle. Bereits kurze Phasen einer fehlenden Oxygenierung haben bei
Kindern dramatische Auswirkungen auf den Sauerstoffgehalt des Blutes. Die im Vergleich zum Erwachsenen deutlich kürzere Apnoetoleranz erklärt sich durch den hohen Sauerstoffbedarf der Kinder mit gleichzeitig niedriger funktioneller Residualkapazität und damit geringeren pulmonalen Sauerstoffspeichern. Die kindliche Atemfrequenz und Atemarbeit ist bereits unter physiologischen Bedingungen altersabhängig erhöht (Tab. 1).

Kinder haben eine verhältnismäßig kleine Mundöffnung mit relativ großer Zunge. Die Epiglottis ist lang und häufig U-förmig

\section{Tabelle 1}

Altersabhängige Maße und Normwerte beim Kind und Empfehlungen zur Beatmung [3].

\begin{tabular}{|c|c|c|c|c|}
\hline Alter & $\begin{array}{l}\text { typisches } \\
\text { Gewicht } \\
\text { (kg) }\end{array}$ & $\begin{array}{l}\text { normale } \\
\text { Herzfrequenz } \\
(1 / \mathrm{min})\end{array}$ & $\begin{array}{l}\text { normale } \\
\text { Atemfrequenz } \\
(1 / \mathrm{min})\end{array}$ & $\begin{array}{l}\text { Beatmungsfrequenz (1/min) } \\
\text { AZV: } 6-10 \mathrm{ml} / \mathrm{kg} \\
\text { Paw } 13-20 \mathrm{mbar} \\
\text { PEEP } 3-5 \mathrm{mbar} \\
\text { I:E } 1: 1-1: 3\end{array}$ \\
\hline FG & $2,5-3,0$ & $130-180$ & $40-60$ & $40-60$ \\
\hline NG & 3,5 & $120-170$ & $40-60$ & $30-40$ \\
\hline 2 Mon. & 4,5 & $120-170$ & $25-40$ & $30-40$ \\
\hline 6 Mon. & 7 & $100-150$ & $25-40$ & $25-30$ \\
\hline 12 Mon. & 10 & $100-150$ & $25-40$ & $25-30$ \\
\hline 18 Mon. & 12 & $100-150$ & $25-40$ & $20-25$ \\
\hline $2 \mathrm{~J}$. & 14 & $80-130$ & $20-30$ & $20-25$ \\
\hline 4J. & 17 & $80-130$ & $20-30$ & $18-22$ \\
\hline $6 \mathrm{~J}$. & 21 & $70-110$ & $20-25$ & $18-22$ \\
\hline $8 \mathrm{~J}$. & 25 & $70-110$ & $20-25$ & $16-20$ \\
\hline $10 \mathrm{~J}$. & 31 & $70-110$ & $20-25$ & $16-20$ \\
\hline $12 \mathrm{~J}$. & 40 & $60-100$ & $12-20$ & $16-20$ \\
\hline
\end{tabular}

gerollt bei gleichzeitig weiter ventral und kranial gelegenem Larynx und Glottis. Bei Manipulation kommt es zudem schnell zu Schleimhautschwellungen, die rasch zu Atemwegsverlegungen führen können.

Cave: Daher sollte jeweils die am wenigsten invasive Technik der Atemwegssicherung gewählt werden. 
Atemwegsmanagement beim Kind

\section{Schritt für Schritt}

\section{Atemwegsmanagement unter erhaltener Spontanatmung}

Atemwegsmanagement bei Kindern kann auch bedeuten, sich in Zurückhaltung zu üben. Vor allem bei erhaltenen Schutzreflexen und vorhandener Spontanatmung kann man bei Bedarf auch bei Kindern ausschließlich großzügig Sauerstoff applizieren, um eine Hypoxämie zu vermeiden.
Praktische Tipps: Bei Atemwegsobstruktion lässt sich durch Anfeuchten der Atemluft häufig eine zügige Besserung der Beschwerden erreichen ggf. in Kombination mit der Verneblung von Adrenalin (z. B. 1-4 mg Adrenalin inhalativ $=0,25-1 \mathrm{ml}$ Infektokrupp ${ }^{\circledR}$ verdünnt auf $5-10 \mathrm{ml} \mathrm{NaCl}$ oder $2,5-5 \mathrm{mg}$ Salbutamol inhalativ verdünnt auf $2-4 \mathrm{ml} \mathrm{NaCl}$ ).

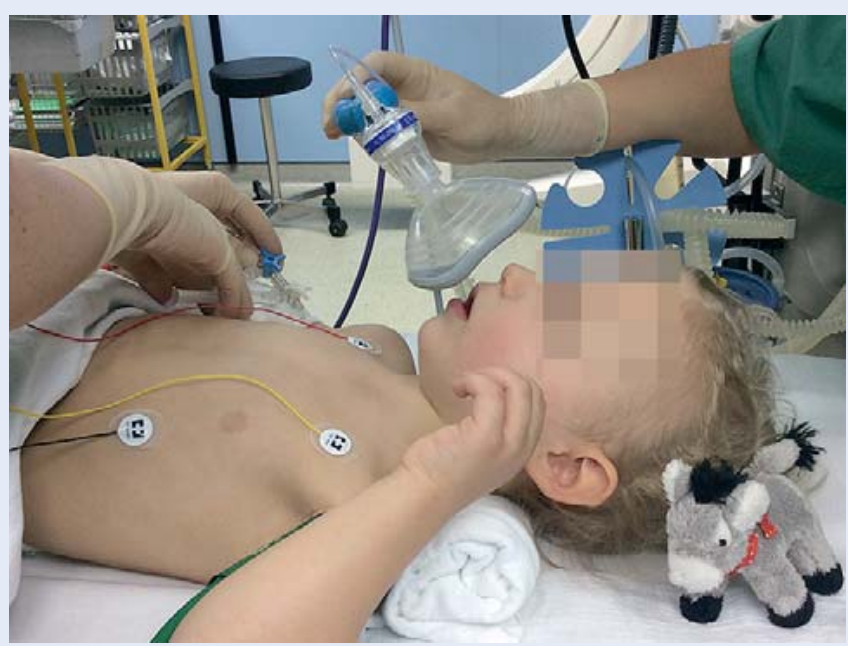

Abb. 1 Bei kleinen Kindern gelingt die Sauerstoffapplikation am besten mit vorgehaltener Sauerstoffmaske bei hohem Frischgasfluss. Bei größeren, kooperativen Kindern können Masken mit Reservoirbeutel die inspiratorische Sauerstoffkonzentration zusätzlich erhöhen.

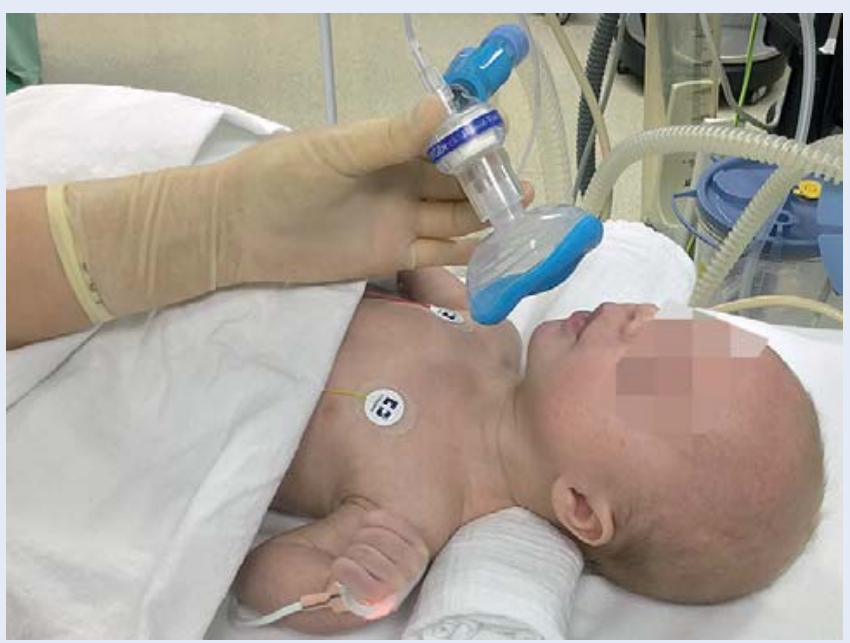

Abb. 2 Um besseren Blickkontakt mit dem Kind halten zu können, kann es günstig sein, die Maske von vorn bzw. neben dem Kind sitzend zu halten. Bei wachen und möglicherweise ängstlichen Kindern bietet es sich auch an, die Kinder auf dem Schoß der Eltern sitzen zu lassen und die Maske zur Sauerstoffapplikation in gegenübersitzender Position zu halten. 


\section{Schritt für Schritt}

\section{Atemwegsmanagement mithilfe von Gesichtsmasken}

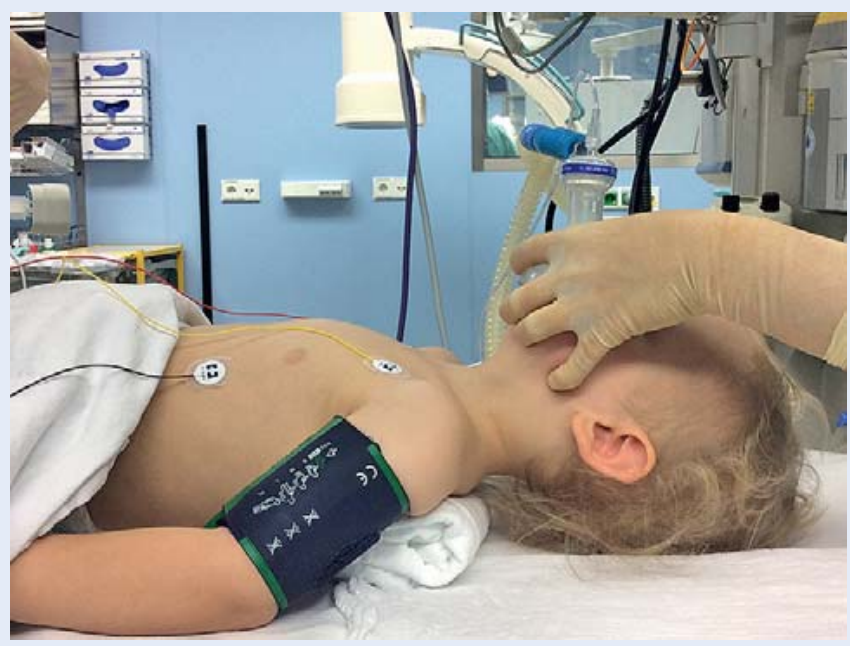

Abb. 3 Für eine erfolgreiche Maskenventilation ist die optimale Lagerung des Kindes obligatorisch. Das Kind sollte mithilfe einer Nackenrolle als Unterlagerung der Schulterregion in Neutralposition (Schnüffelstellung) gebracht werden (Cave: großen Kopf des Kindes beachten), um eine Verlegung des Atemwegs durch Zurückfallen der großen Zunge auf die Rachenhinterwand zu verhindern.

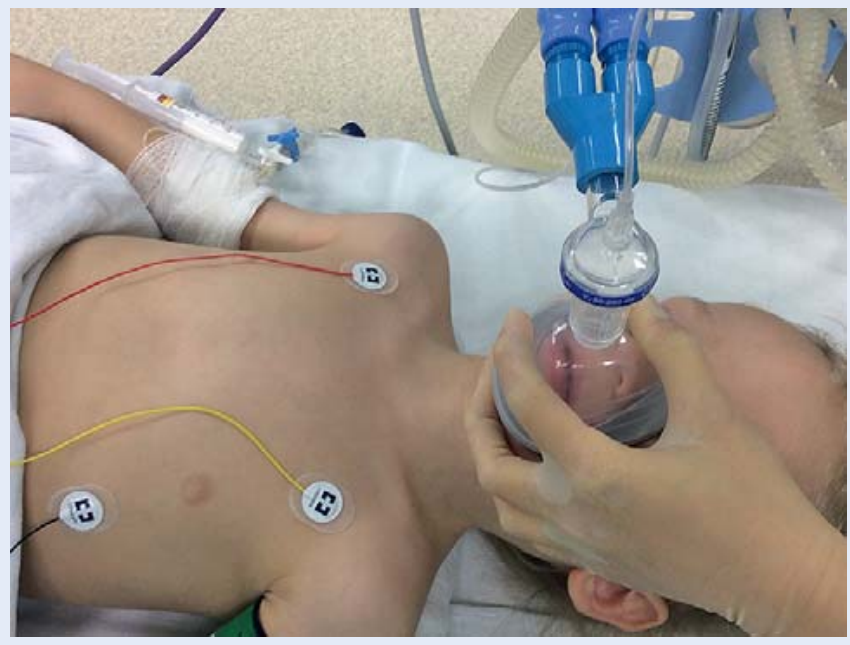

Abb. 5 Die Auswahl der geeigneten Gesichtsmaske erfolgt altersabhängig. Bei Früh- bzw. Neugeborenen eignen sich v. a. runde, durchsichtige Gesichtsmasken aus Silikon, bei Säuglingen auch ovale Masken. Wichtig bei der Auswahl der geeigneten Maske ist, dass diese dicht sitzend Mund- und Nasenöffnung umschließt, ohne über Kinn- und Augenbereich hinauszuragen.

Cave: Um eine Luftinsufflation in den Magen mit konsekutivem Zwerchfellhochstand, Atelektasenbildung und Verschlechterung der Oxygenierung zu vermeiden, sollte man den Beatmungsspitzendruck möglichst $<15$ mbar halten. Bei bereits erfolgter Magenüberblähung sollte der Magen mithilfe einer Magensonde oder eines gastral platzierten Absaugkatheters abgesaugt und entlastet werden [5].

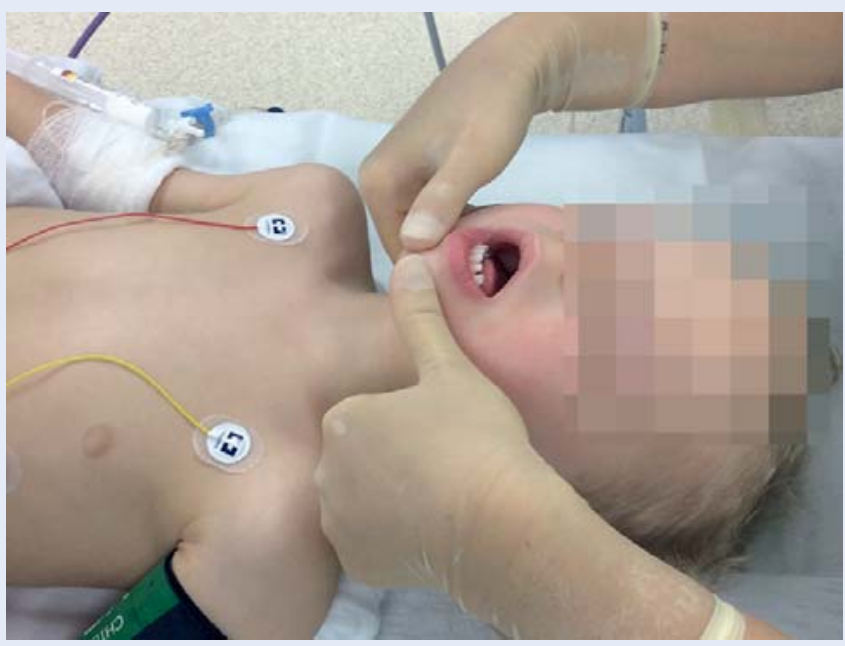

Abb. 4 Bei nicht mehr suffizienter Spontanatmung ist der sofortige Beginn der Beatmung mittels Gesichtsmaske indiziert, um eine Hypoxämie und deren kardiale Folgen zu vermeiden. Mittels Protrusion wird nun der Unterkiefer nach vorne gezogen und der Mund gleichzeitig geöffnet. Dabei ist darauf zu achten, dass die Zunge nicht oben am Gaumen anliegt, sondern nach unten fällt und im Unterkiefer zu liegen kommt - dann erst erfolgt das Aufsetzen der Maske. Eine durchsichtige Gesichtsmaske hilft dabei, während der Maskenbeatmung ein kontinuierliches optisches Feedback der korrekten Position zu erhalten.

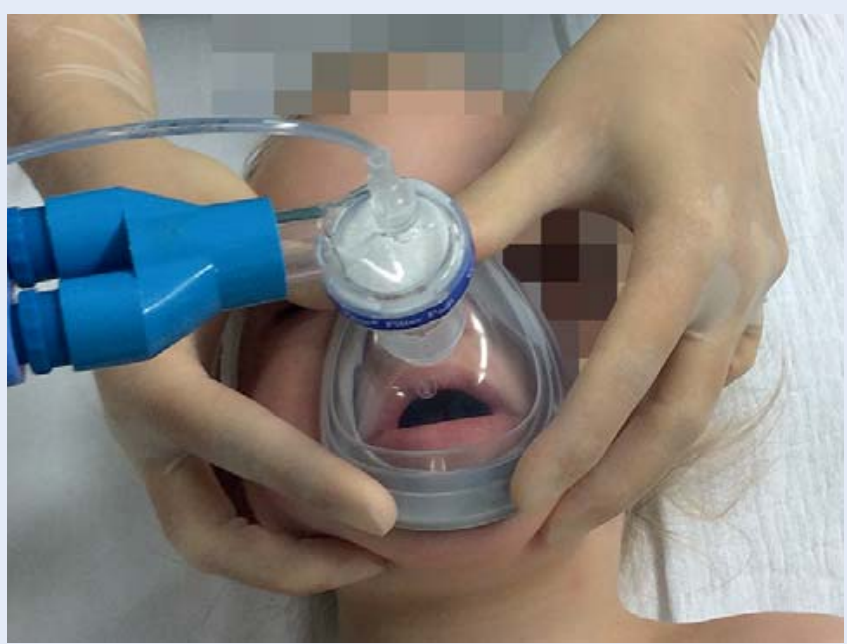

Abb. 6 Beim Erwachsenen häufig eingesetzte Atemwegshilfen für die Erleichterung der Maskenbeatmung, wie Guedel- oder Wendl-Tuben, sind auch in verschiedenen Kindergrößen erhältlich. Diese sollten beim Kind aber erst zur Anwendung kommen, wenn eine Beatmung mittels Gesichtsmaske trotz der oben beschriebenen Lagerungstechniken weiterhin erschwert ist, da jede Manipulation im kindlichen Naso- und Oropharynx neben Schleimhautschwellung und Blutung bei mangelnder Sedierung auch zum Laryngospasmus führen kann [4].

Praktische Tipps: Eine Zwei-Helfer-Methode erleichtert die Maskenbeatmung im Kindesalter, v.a. bei fehlender Routine beim kindlichen Atemwegsmanagement. 


\section{Schritt für Schritt}

\section{Atemwegsmanagement mithilfe von supraglottischen Atemwegshilfen}

Gestaltet sich die Maskenbeatmung schwierig, besteht die nächste Stufe der Eskalation im Einsatz von supraglottischen Atemwegshilfen (SGA) [6]. Prinzipiell sind hier die Larynxmaske (LM) oder der Larynxtubus (LT) verfügbar. Die erfolgreiche Anwendung des LT bei Kindern beschränkt sich derzeit auf wenige Arbeitsgruppen und Zentren, die mutmaßlich über eine entsprechende Expertise bezüglich der routinemäßigen Anwendung des LT verfügen. Unter Berücksichtigung der wissenschaftlichen Datenlage und der großen klinischen Erfahrung mit der LM in der Elektiv- und Notfallanwendung bei Kindern wird derzeit zum alternativen Atemwegsmanagement im Kindesalter primär die LM empfohlen. Vor allem für Kinder unter $10 \mathrm{~kg}$ Körpergewicht erscheint der Einsatz des LT aufgrund der aktuellen Studienlage nicht sicher durchführbar und wird daher derzeit nicht empfohlen [7]. Die LM sollte präklinisch daher in allen verfügbaren Größen (1-5) vorgehalten und ihre Anwendung regelmäßig geschult werden.

Die meisten Hersteller von SGA bieten mittlerweile Modelle an, die einen Drainagekanal integriert haben. Diese sog. SGA der 2. Generation ermöglichen somit eine zuverlässige Trennung von Gastrointestinal- und Respirationstrakt. Vor allem für die Notfallversorgung erscheint dies sinnvoll, da gastrale Sekrete und Luft nicht nur passiv über den Drainagekanal abfließen können, sondern sich durch die Insertion einer Magensonde auch aktiv evakuieren lassen. Das Konzept der LM zur Verwendung bei Kindern unterscheidet sich nicht zur Verwendung bei Erwachsenen. Weiterführende Informationen zu Anwendung und Funktion von LM der 2. Generation finden sich auch bei $[8,9]$.

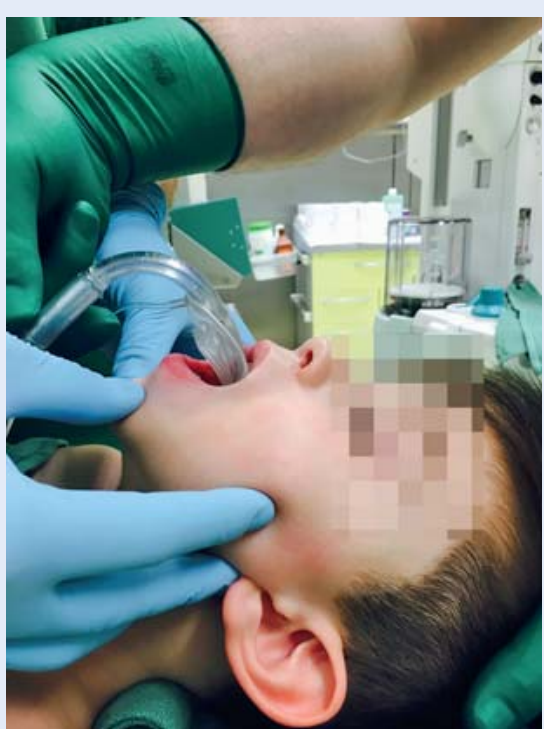

Abb. 7 Die Auswahl der geeigneten LM erfolgt gewichts- bzw. größenabhängig (Tab. 2). Zur Platzierung wird die LM entblockt und entlang des harten Gaumens vorgeschoben. Dafür wird der Kopf des Kindes mittels Nackenrolle und Positionierung in Neutralposition gelagert. Eine Unterkieferprotrusion mit gleichzeitiger Mundöffnung erleichtert die Insertion.

\section{Tabelle 2}

Altersabhängige Empfehlungen zur Auswahl von Larynxmaske und Endotrachealtubus (geblockt) [10].

\begin{tabular}{|c|c|c|c|}
\hline Alter & $\begin{array}{l}\text { Larynxmaske } \\
\text { (Größe) }\end{array}$ & $\begin{array}{l}\text { Tubus-ID } \\
\geq 1 \mathrm{a}: \\
\text { Alter/4 + } 4\end{array}$ & $\begin{array}{l}\text { Tubustiefe oral } \\
\text { ab Zahnleiste }(\mathrm{cm}) \\
\geq 1 \mathrm{a}: \\
\text { Alter/2+12 }\end{array}$ \\
\hline FG & 1 & $2,5-3,0$ & $6+\mathrm{kgKG}$ \\
\hline NG & 1 & 3,5 & $9-10$ \\
\hline 2 Mon. & 1 & 3,5 & $10-11$ \\
\hline 6 Mon. & $11 / 2$ & 3,5 & 11 \\
\hline 12 Mon. & $1 \frac{1}{2}$ & 4,0 & 12 \\
\hline 18 Mon. & 2 & 4,5 & 13 \\
\hline $2 \mathrm{~J}$. & 2 & 4,5 & 13 \\
\hline $4 \mathrm{~J}$. & 2 & 5,0 & 14 \\
\hline $6 \mathrm{~J}$. & $21 / 2$ & 5,5 & 15 \\
\hline $8 \mathrm{~J}$. & $21 / 2$ & 6,0 & 16 \\
\hline $10 \mathrm{~J}$. & 3 & 6,5 & 18 \\
\hline $12 \mathrm{~J}$. & 3 & 7,0 & 20 \\
\hline
\end{tabular}

Praktische Tipps: Befindet sich die Herstellerangabe zur Größenauswahl genau an der Grenze zwischen 2 Maskengrößen, so empfiehlt es sich, die größere Maske zu wählen. Um eine sichere Anwendung bei möglichst geringer Atemwegsmorbidität zu gewährleisten, sollte der Cuff-Druck einen Wert von 60 mbar nicht überschreiten. 


\section{Atemwegsmanagement beim Kind}

\section{Schritt für Schritt}

\section{Atemwegsmanagement mithilfe der endotrachealen Intubation}

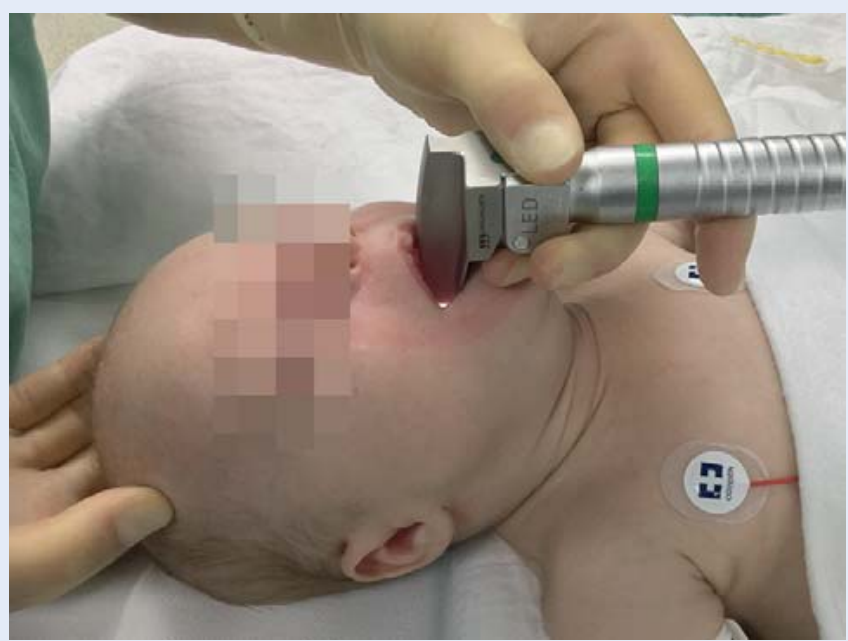

Abb. 9 Die endotracheale Intubation bleibt auch (oder gerade besonders) in Notfallsituationen einem in der Anwendung bei Kindern erfahrenen Mediziner vorbehalten. Die Auswahl des geeigneten Endotrachealtubus erfolgt alters- bzw. gewichtsabhängig (Tab. 2). Hierbei sollten blockbare Tuben mit Low-PressureCuffs und Cuff-Druck-Messung (max. 20 mbar) bevorzugt eingesetzt werden [11]. Spatelgröße und korrekte Tubustiefe sind ebenfalls altersabhängig zu wählen.

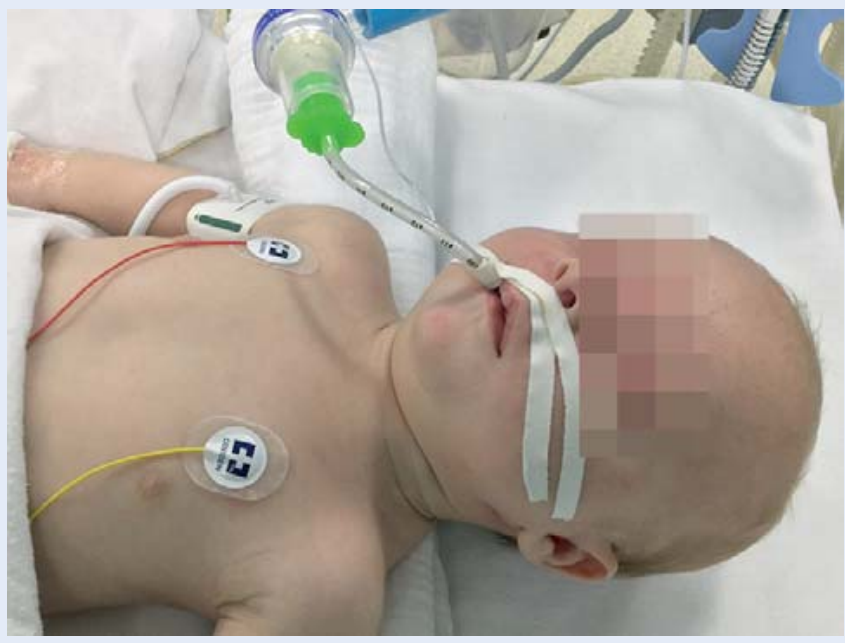

Abb. 11 Nach erfolgreicher Intubation ist eine gute und exakte Fixierung des Endotrachealtubus unerlässlich, da bereits Veränderungen der Tubuslage von weniger als $1 \mathrm{~cm}$, z. B. durch Kopfbewegungen oder Lageveränderungen beim Säugling, zwischen einseitiger Intubation oder versehentlicher Extubation entscheiden können.

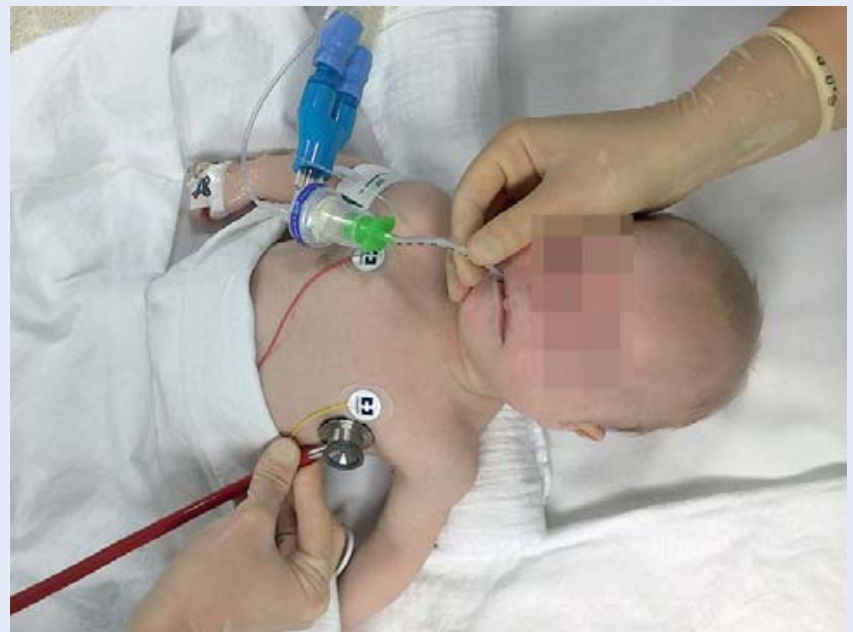

Abb. 10 Eine ausreichende Narkosetiefe ohne erhaltene Spontanatmung und der zusätzliche Einsatz von Muskelrelaxanzien erleichtert die Intubation in der Notfallsituation [4]. Auskultatorische Lagekontrolle und kontinuierliche Kapnografie sind obligatorisch.

Praktische Tipps: Der 2. Helfer kann durch eine vorsichtige externe Manipulation auf Höhe des Schildknorpels (z. B. Rechtsverschiebung des Larynx) möglicherweise eine verbesserte Sicht auf den Larynx erreichen.

Cave: Die Kapnografie im Rettungsdienst ist bei jeder invasiven Form der Atemwegssicherung zwingend erforderlich, da nur so eine korrekte Lage der SGA bzw. eine endotracheale Tubuslage mit suffizienter Ventilation sichergestellt werden kann. Die Kapnometrie erfolgt bei den transportablen Beatmungsgeräten im Rettungsdienst i.d. R. mittels zwischengeschalteter Messküvette im Hauptstromverfahren. Bei Säuglingen sind, bedingt durch die kleinen Atemzugvolumina, falsch niedrig gemessene $\mathrm{CO}_{2}$-Werte möglich, daher sollte sich die Einstellung der Beatmungsparameter nicht nur am endexspiratorischen $\mathrm{CO}_{2}$, sondern auch an den Empfehlungen zur Beatmungseinstellung (Tab. 1) orientieren. 
Interessenkonflikt: Die Autoren geben an, dass kein Interessenkonflikt besteht.

\section{Literatur}

1 Eich C, Russo SG, Heuer JF et al. Characteristics of out-of-hospital paediatric emergencies attended by ambulance- and helicopter-based emergency physicians. Resuscitation 2009; 80: 888-892

2 Eich C, Roessler M, Nemeth M et al. Characteristics and outcome of prehospital paediatric tracheal intubation attended by anaesthesia-trained emergency physicians. Resuscitation 2009; 80: 13711377

3 Coté CJ, Lerman J, Anderson BJ. A Practice of Anesthesia for Infants and Children. In: Marciniak B,

Hrsg. Growth and Development. 5th ed. Amsterdam: Elsevier; 2013

4 Russo SG, Trieschmann U, Nicolai T. Atemwegsmanagement bei Kindern in Notfallsituationen. Notfall Rettungsmed 2014; 17: 105-112

5 Wenzel V, Idris AH, Banner MJ et al. Respiratory system compliance decreases after cardiopulmonary resuscitation and stomach inflation: impact of large and small tidal volumes on calculated peak airway pressure. Resuscitation 1998; 38: 113-118

6 Timmermann A, Russo SG, Crozier TA et al. Novices ventilate and intubate quicker and safer via intubating laryngeal mask than by conventional bagmask ventilation and laryngoscopy. Anaesthesiology 2007; 107: 570-576

7 Keil J, Jung P, Schiele A et al. Interdisziplinär konsentierte Stellungnahme zum Atemwegsmanagement mit supraglottischen Atemwegshilfen in der Kindernotfallmedizin. Anaesthesist 2015; DOI: 10.1007/s00101-015-0107-6

8 Timmermann A, Bergner UA, Russo SG. Laryngeal mask airway indications: new frontiers for secondgeneration supraglottic airways. Curr Opin Anaesthesiol 2015; 28: 717-726

9 Russo SG, Wulf H. Erweiterte Indikationen der Larynxmaske - Wo liegen die Limitationen? Anästhesiol Intensivmed Notfallmed Schmerzther 2014; 49: 152-161

10 Coté CJ, Lerman J, Anderson BJ. A Practice of Anesthesia for Infants and Children. In: Litman RS, Fiadjou JE, Stricker PA et al., Hrsg. The Pediatric Airway. 5th ed. Amsterdam: Elsevier; 2013

11 Weiss M, Dullenkopf A, Fischer JE et al. Prospective randomized controlled multi-centre trial of cuffed or uncuffed endotracheal tubes in small children. $\mathrm{Br}$ J Anaesth 2009; 103: 867-873

\section{Über die Autoren}

\section{Nora Jahn}

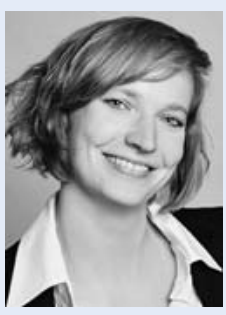

Arbeitet an der Klinik und Poliklinik für Anästhesiologie und Intensivtherapie des Universitätsklinikums Leipzig als Fachärztin für Anästhesie mit der Zusatzbezeichnung Notfallmedizin. Ihre Forschungs-

schwerpunkte liegen im Bereich der angewandten Physiologie.

E-Mail: nora.jahn@medizin.uni-leipzig.de

\section{Sebastian G. Russo}

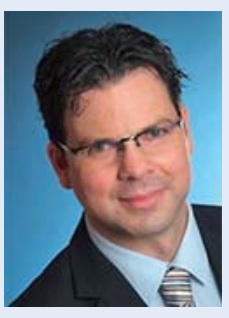

Arbeitet an der Klinik für Anästhesiologie der Universitätsmedizin Göttingen. Sein klinisch-wissenschaftliches Interesse gilt den modernen Strategien der Beatmung und Atemwegssicherung sowie der außer- und innerklinischen Notfallversorgung.

\section{Claudia Höhne}

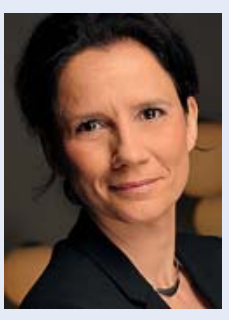

Arbeitet an der Klinik und Poliklinik für Anästhesiologie und Intensivtherapie des Universitätsklinikums Leipzig als Geschäftsführende Oberärztin. Ihre Forschungsschwerpunkte liegen im Bereich der Kinderanästhesie und der angewandten Physiologie.

E-Mail: claudia.hoehne@medizin.uni-leipzig.de

\section{Korrespondenzadresse}

Dr. med. Nora Jahn

Klinik und Poliklinik für Anästhesiologie und Intensivtherapie

Universitätsklinikum Leipzig AöR

Liebigstr. 20

04103 Leipzig

E-Mail: nora.jahn@medizin.uni-leipzig.de

Koordination der Rubrik „Schritt für Schritt“:

PD Dr. med. Michael Bernhard, Leipzig 